\title{
Sonic hedgehog signaling in hepatocellular carcinoma: A pilot study
}

\author{
MOHANNAD DUGUM ${ }^{1}$, IBRAHIM HANOUNEH ${ }^{2}$, THOMAS MCINTYRE $^{3}$, \\ RISH PAI $^{4}$, FEDERICO AUCEJO 5 , BIJAN EGHTESAD 5 and NIZAR ZEIN ${ }^{2}$ \\ Departments of ${ }^{1}$ Internal Medicine, Medicine Institute, ${ }^{2}$ Gastroenterology and Hepatology, Digestive Disease Institute, \\ ${ }^{3}$ Cellular and Molecular Medicine, Lerner Research Institute and ${ }^{4}$ Pathology, Pathology and Laboratory Medicine Institute; \\ ${ }^{5}$ Transplantation Center, Digestive Disease Institute, Cleveland Clinic, Cleveland, OH 44195, USA
}

Received May 20, 2015; Accepted October 6, 2015

DOI: $10.3892 / \operatorname{mco} .2016 .728$

\begin{abstract}
Hedgehog signaling is involved in the pathogenesis of several tumor types; however, its role in hepatocellular carcinoma (HCC) has not been fully elucidated. Biomarkers that reflect tumor aggressiveness are of potential value in selecting HCC patients for liver transplantation (LT). The aim of the present study was to assess the tissue expression of sonic hedgehog (Shh) biomarkers in HCC and surrounding non-tumorous liver tissue, and to correlate this expression with HCC recurrence following LT. Patients who underwent LT for HCC at the Cleveland Clinic (Cleveland, OH, USA) between 2002 and 2006 were randomly selected for analysis. Tissue samples were retrieved from the explanted tumorous livers. Routine immunohistochemistry was used to detect three specific Shh pathway biomarkers: The ligand Shh, the receptor patched-1 (Ptch) and the transcription factor glioma-associated oncogene homolog 1 (Gli1). Computerized quantitative analysis was used to evaluate the expression levels of these markers in HCC and surrounding non-tumorous liver tissue. Analysis of variance was used to compare the differential tissue expression between patients with and those without HCC recurrence. A time-to-event analysis was performed to assess the association of hedgehog biomarker expression with the risk of HCC recurrence following LT. A total of 53 tissue specimens from 21 patients were analyzed. The mean patient age was $57 \pm 8$ years and $86 \%$ of the patients were male. A total of $62 \%$ patients had hepatitis $\mathrm{C}$ virus infection, $14 \%$ had hepatitis B virus infection, $43 \%$ had alcoholic cirrhosis and $91 \%$ fulfilled the Milan criteria at the time of LT. The average follow-up time after LT was $36 \pm 15$ months, during which $19 \%$ of the patients developed HCC recurrence and $29 \%$ died.
\end{abstract}

Correspondence to: Dr Mohannad Dugum, Department of Internal Medicine, Medicine Institute, Cleveland Clinic, 9500 Euclid Avenue - NA10, Cleveland, OH 44195, USA

E-mail: mdugum.md@gmail.com

Key words: liver cancer, hedgehog, biomarkers, liver transplantation, tumor recurrence
Shh, Ptch and Gli1 were detected in the HCC tissues of all the patients. Ptch was overexpressed in HCC compared with the surrounding non-tumorous tissue. The statistical power of this study was unable to associate Shh pathway markers with HCC recurrence following LT. In a proof-of-concept study, we demonstrated tissue expression of three Shh biomarkers within HCC tumors, and also identified differences in Ptch expression between tumor and surrounding non-tumorous tissue. Further larger studies are required to assess the utility of these biomarkers in HCC.

\section{Introduction}

The hedgehog signaling pathway plays a pivotal role in embryogenesis and is involved in the regulation of cell growth and differentiation (1). There are 3 hedgehog ligands, among which sonic hedgehog (Shh) is the best characterized. The hedgehog pathway is activated by binding of one of those 3 ligands to the receptor patched-1 (Ptch). Unbound Ptch acts as a tumor suppressor that binds to and represses the proto-oncoprotein smoothened (Smo), thereby preventing it from activating downstream transcription factors, particularly the glioma-associated oncogene homolog 1 (Gli1). Activation of hedgehog signaling has been demonstrated to be a key factor in the development and progression of a number of human malignancies, including skin, brain and gastrointestinal cancers (2). The malignancies in which hedgehog signaling has been implicated also include hepatocellular carcinoma (HCC) $(3,4)$.

With $>700,000$ newly diagnosed cases annually, HCC represents a major global health burden (5-7). In carefully selected patients, liver transplantation (LT) represents the most effective treatment for $\operatorname{HCC}(8,9)$. Currently, criteria based on the number and size of HCC lesions are used to select HCC patients for LT, referred to as Milan criteria. However, a significant shortcoming of these criteria is defining the risk of HCC recurrence following LT based on tumor morphology rather than biology. In a study from our institution, we demonstrated that using $\mathrm{HCC}$ volume as a static value inaccurately reflects HCC tumor behavior (10). Indeed, among patients with a tumor burden beyond the currently adopted Milan criteria, there is a subset with favorable tumor biology that may have positive outcomes if transplanted. 
Combining novel tumor biomarkers with conventional clinical indicators of prognosis should more accurately predict HCC patient outcomes, enabling more appropriate therapeutic decisions. The aim of this study was to investigate the tissue expression patterns of Shh biomarkers in HCC and associate their expression with the risk of HCC recurrence following LT.

\section{Patients and methods}

Study population. Adult patients diagnosed with stage T2 HCC who underwent LT from cadaveric donors at the Cleveland Clinic between January 1, 2002 and December 31, 2006 were randomly selected for analysis. Demographic patient data, including underlying liver disease (hepatitis $\mathrm{B}$ and/or $\mathrm{C}$ virus infection, alcoholic liver disease and non-alcoholic steatohepatitis), laboratory values, post-LT immunosuppression regimen, imaging studies and liver explant pathology reports, were extracted from the electronic medical database. Pathological tissue specimens of HCCs and surrounding non-tumorous liver tissue were retrieved from the explanted livers. All the transplanted patients were regularly monitored following LT under the Cleveland Clinic liver transplant service protocols: $\mathrm{CT}$ or MRI scans of the chest, abdomen and pelvis were performed every 3 months during the first year after LT, every 6 months during the second year, and yearly thereafter. HCC recurrence following LT was defined as months from LT to event or censoring. The subjects were censored at the time of re-transplantation or last follow-up. The patients were then grouped according to HCC recurrence following LT.

Immunohistochemical analysis. Routine immunohistochemistry was used to evaluate the different hedgehog pathway proteins in explanted liver tissue (in tumor as well as in the surrounding non-tumorous liver parenchyma). Briefly, paraffin-embedded tissue sections were deparaffinized, using SAFE-CLEAR Clearant (EK Industries, Inc., Joliet, IL, USA), which is a xylene substitute, as the tissue clearing agent. Ethanol hydration and serial dilution were performed, followed by phosphate-buffered saline (PBS) washout. Diluted sodium citrate buffer was then used to heat the specimens. After cooling, donkey serum (10\%) was added for $30 \mathrm{~min}$, followed by overnight incubation at $4^{\circ} \mathrm{C}$ with primary antibodies targeting Shh, Ptch and Gli1; these were goat polyclonal anti-Shh antibody (dilution, 1:100; N-19, sc-1194), rabbit polyclonal anti-Ptch antibody (dilution, 1:200; H-267, sc-9016) and goat polyclonal anti-Gli1 antibody (dilution, 1:100; N-16, sc-6153), all from Santa Cruz Biotechnology, Inc., Dallas, TX, USA. Matched negative controls were stained without the primary antibodies. PBS washout was repeated, then secondary fluorescent antibodies were applied for $60 \mathrm{~min}$; these were donkey anti-goat IgG-CFL 488 (dilution, 1:100; sc-362255) or donkey anti-rabbit IgG-CFL 488 (dilution, 1:100; sc-362261), both from Santa Cruz Biotechnology, Inc. PBS and then water washout were repeated. Finally, mounting was performed with 4',6-diamidino-2-phenylindole as the nuclear stain.

The presence and abundance of the three hedgehog pathway proteins in all the specimens was evaluated using computerized quantitative software analysis with the aid of an experienced hepatopathologist. The tissue expression level of
Table I. Patient characteristics $(\mathrm{n}=21)$.

\begin{tabular}{lc}
\hline Variables & Values \\
\hline Age (years) & $56.6 \pm 8.0$ \\
Male & $18(85.7)$ \\
BMI $\left(\mathrm{kg} / \mathrm{m}^{2}\right)$ & $31.6 \pm 4.0$ \\
Caucasian & $18(85.7)$ \\
Hepatitis C virus infection & $13(61.9)$ \\
Hepatitis B virus infection & $3(14.3)$ \\
Alcoholic liver disease & $9(42.9)$ \\
Non-alcoholic steatohepatitis & $1(4.8)$ \\
Pre-LT $\alpha$-fetoprotein & $85.7 \pm 179.5$ \\
Radiology, number of nodules & \\
0 & $5(23.8)$ \\
1 & $9(42.9)$ \\
2+ & $7(33.3)$ \\
Radiology, within Milan criteria & $19(90.5)$ \\
Radiology, within UCSF criteria & $20(95.2)$ \\
Pathology, number of nodules & \\
1 & $14(66.7)$ \\
2+ & $7(33.3)$ \\
Pathology, within Milan criteria & $21(100.0)$ \\
Microvascular invasion & $6(28.6)$ \\
Grade & \\
Well-differentiated & $9(42.9)$ \\
Moderately-poorly differentiated & $12(57.1)$ \\
Biochemical MELD at the time of LT & $15.4 \pm 5.6$ \\
Shh expression level & \\
Tumor & $126.2 \pm 30.2$ \\
Cirrhotic tissue & $130.7 \pm 32.4$ \\
Gli1 expression level & \\
Tumor & $5.6 \pm 0.88$ \\
Cirrhotic tissue & $5(28.6)$ \\
Ptch expression level & $5.8 \pm 0.93$ \\
Tumor & \\
Pirrhotic tissue & $7.0 \pm 0.90$ \\
HCC recurrence & \\
\hline
\end{tabular}

Values are presented as mean \pm standard deviation or no. (\%). BMI, body mass index; UCSF, University of California San Francisco; LT, liver transplantation; MELD, model for end-stage liver disease; Shh, sonic hedgehog; Gli1, glioma-associated oncogene homolog 1; Ptch, receptor patched-1; HCC, hepatocellular carcinoma.

hedgehog biomarkers in each HCC nodule was correlated with HCC recurrence and disease-free survival following LT.

Statistical analysis. Continuous variables are expressed as mean \pm standard deviation and categorical variables as no. (\%). 

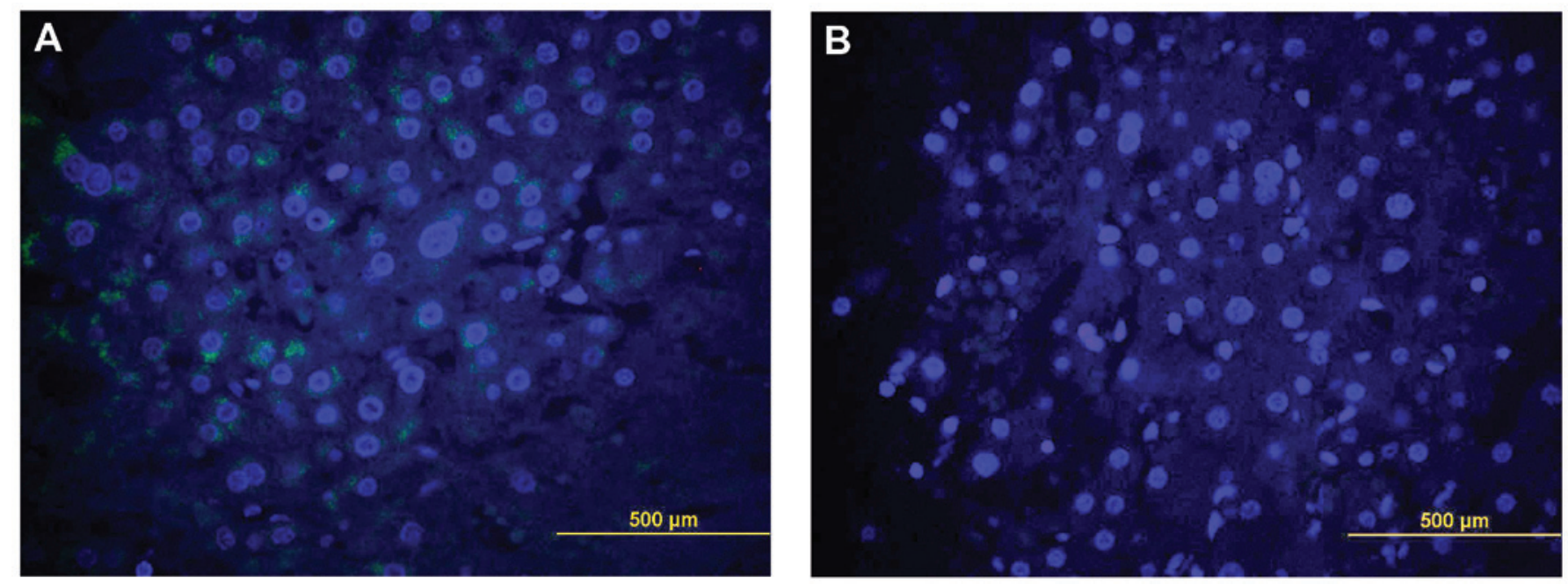

Figure 1. (A) Cytoplasmic expression of sonic hedgehog protein in hepatocellular carcinoma cells as demonstrated by green immunoflurescent antibodies. (B) Negative control section of hepatocellular carcinoma cells without the immunoflurescent antibodies. The nuclei were stained with 4',6-diamidino-2-phenylindole (blue fluorescence).
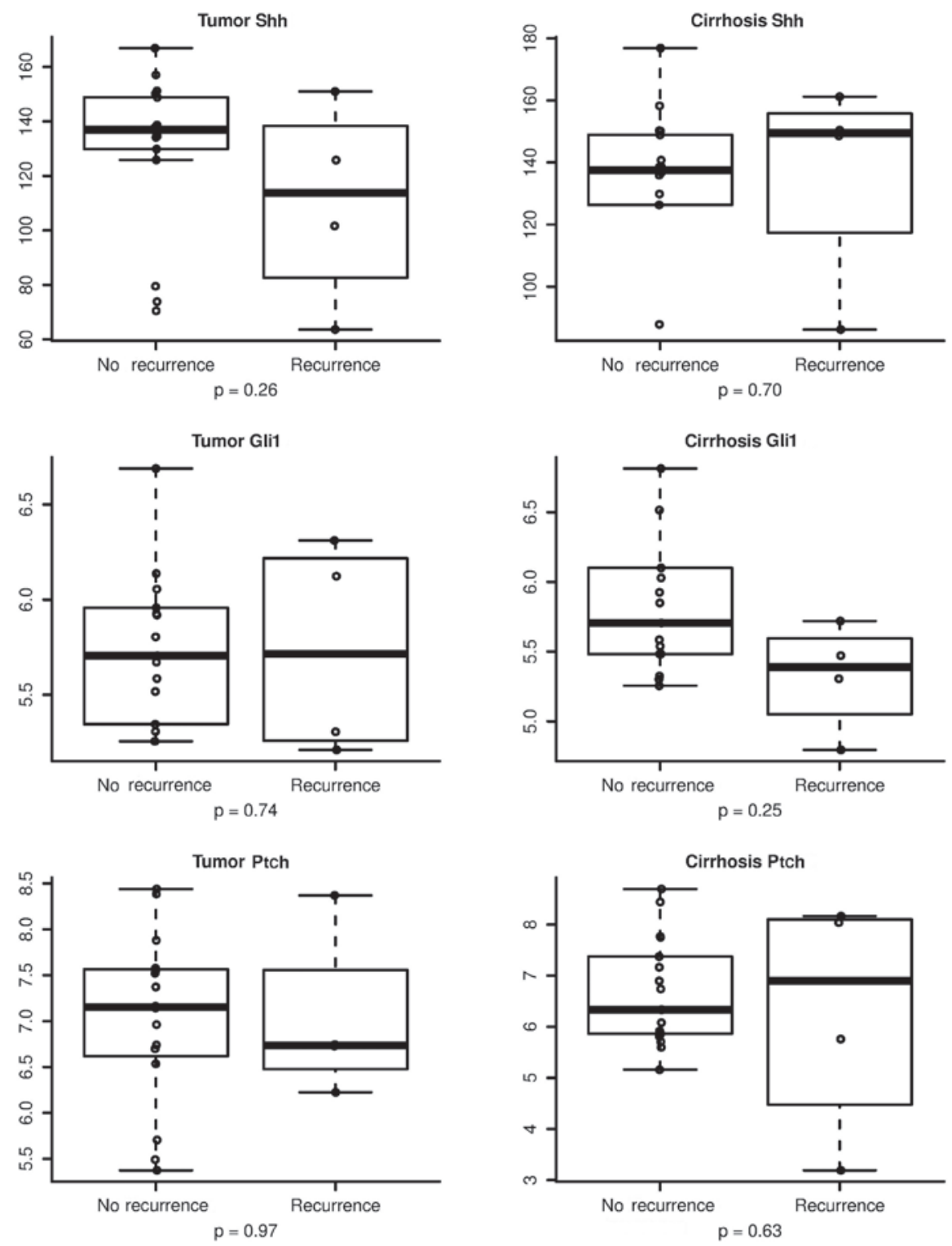

Figure 2. Association of post-liver transplantation hepatocellular carcinoma recurrence with the expression levels of Shh, Ptch and Gli1 in the tumor and surrounding non-tumorous liver tissues. Shh, sonic hedgehog; Ptch, receptor patched-1; Gli1, glioma-associated oncogene homolog 1. 
Table II. Association of HCC recurrence with Shh biomarkers.

\begin{tabular}{|c|c|c|c|}
\hline Biomarkers & $\begin{array}{l}\text { No HCC recurrence } \\
\text { (tissue samples, } n=42 ; \\
\text { patients, } n=17 \text { ) }\end{array}$ & $\begin{array}{c}\text { HCC recurrence } \\
\text { (tissue samples, } n=11 ; \\
\text { patients, } n=4 \text { ) }\end{array}$ & P-value \\
\hline \multicolumn{4}{|l|}{ Shh } \\
\hline Tumor & $129.9 \pm 28.4$ & $110.5 \pm 37.2$ & 0.26 \\
\hline Cirrhotic tissue & $129.3 \pm 32.9$ & $136.6 \pm 34.1$ & 0.70 \\
\hline \multicolumn{4}{|l|}{ Gli1 } \\
\hline Tumor & $5.6 \pm 0.95$ & $5.7 \pm 0.56$ & 0.74 \\
\hline Cirrhotic tissue & $5.9 \pm 0.99$ & $5.3 \pm 0.39$ & 0.25 \\
\hline \multicolumn{4}{|l|}{ Ptch } \\
\hline Tumor & $7.0 \pm 0.92$ & $7.0 \pm 0.93$ & 0.97 \\
\hline Cirrhotic tissue & $6.7 \pm 1.06$ & $6.3 \pm 2.3$ & 0.63 \\
\hline
\end{tabular}

Values are presented as mean \pm standard deviation with analysis of variance. HCC, hepatocellular carcinoma; Shh, sonic hedgehog; Gli1, glioma-associated oncogene homolog 1; Ptch, receptor patched-1.

Table III. Univariable Cox regression analysis of the association between Shh biomarkers and HCC recurrence.

\begin{tabular}{lcc}
\hline Biomarkers & Hazard ratio $(95 \% \mathrm{CI})$ & P-value \\
\hline Shh & $0.98(0.95-1.01)$ & 0.16 \\
Tumor & $1.01(0.97-1.05)$ & 0.64 \\
Cirrhotic tissue & $1.2(0.35-3.9)$ & \\
Gli-1 & $0.49(0.16-1.5)$ & 0.82 \\
Tumor & $0.79(0.25-2.5)$ & 0.22 \\
Cirrhotic tissue & $0.68(0.32-1.5)$ & 0.69 \\
Ptch & & 0.34 \\
Tumor & \\
Cirrhotic tissue &
\end{tabular}

Shh, sonic hedgehog; HCC, hepatocellular carcinoma; CI, confidence interval; Gli-1, glioma associated oncogene homolog 1; Ptch, receptor patched-1.

Analysis of variance was used to assess differences in tissue expression of each Shh biomarker between HCC patients with and those without tumor recurrence following LT. In addition, a time-to-event analysis was performed to assess whether any of the hedgehog biomarkers was associated with the risk of recurrence of HCC following LT. A P-value of $<0.05$ was considered to indicate statistically significant differences. All analyses were performed with SAS software, version 9.3 (SAS Institute, Cary, NC, USA) and R software, version 3.0.2 (R Foundation for Statistical Computing, Vienna, Austria).

\section{Results}

Expression of Shh, Ptch and Gli1 proteins in HCC samples. A total of 53 tissue samples from 21 patients were included in the analysis. The tissue samples included HCC nodules $(n=32)$ and surrounding non-tumorous cirrhotic liver $(n=21)$. The mean age of the patients was $57 \pm 8$ years, $86 \%$ of the patients were male, $86 \%$ were Caucasian and their mean calculated model for end-stage liver disease (MELD) score was 15. A total of $62 \%$ of the patients had hepatitis $\mathrm{C}$ virus infection, $14 \%$ had hepatitis $B$ virus infection and $43 \%$ had alcoholic cirrhosis (a proportion of the patients had multiple underlying liver diseases). A total of $91 \%$ of the patients had HCCs within the Milan criteria at the time of LT based on radiological assessment, while all the tumors were eventually determined to be within the Milan criteria based on pathological examination following transplantation. The average follow-up time after LT was $36 \pm 15$ months, during which $19 \%$ of the patients developed recurrence of HCC and 29\% died (Table I). Shh, Ptch and Gli1 were detected in the liver tissues of all the patients, within the tumor itself, as well as in the surrounding non-tumorous cirrhotic tissues (Fig. 1). Ptch was overexpressed in HCC compared with the surrounding non-tumorous liver tissue.

Association of Shh, Ptch and Glil expression with HCC recurrence following $L T$. There was no evidence suggesting that any of the hedgehog biomarkers was significantly associated with HCC recurrence following LT (Fig. 2). However, the time-to-event analysis demonstrated a trend toward statistical significance for correlating Shh levels with HCC recurrence following LT. These data indicate that a larger sample size to enhance statistical power is required to confirm the HCC recurrence results. Based on recurrence of HCC following LT, Table II shows the association of HCC recurrence with each hedgehog biomarker and Table III summarizes the hazard ratio of HCC recurrence based on the expression of the hedgehog biomarkers.

\section{Discussion}

LT is currently the standard of care for HCC patients with stage T2 tumors. The United Network for Organ Sharing has adopted the restrictive Milan criteria to select patients with HCC for LT. Based on preoperative imaging, the Milan criteria limit LT to patients with a solitary $\mathrm{HCC}$ tumor $<5.0 \mathrm{~cm}$ in 
diameter, or 2-3 HCC tumors $<3.0 \mathrm{~cm}$ each. The 4-year survival rate of HCC patients with tumors within the Milan criteria is $\sim 85 \%$ following LT (11). However, a serious complication of LT for HCC is tumor recurrence. Even within the Milan criteria, HCC recurrence rates following LT have been found to be as high as $15 \%(12,13)$. In an era of organ shortage, organ allocation policies are crucial to avoid graft misutilization. Subsequently, tumor biomarkers that help characterize HCC biology are sought to determine the most convenient therapeutic options for these patients.

In this study, we evaluated the tissue expression of several components within the hedgehog pathway in HCC tumors, and attempted to determine whether the level of tissue expression correlates with the aggressiveness of tumor behavior in terms of tumor recurrence following LT. The hedgehog pathway is a highly preserved cellular signaling pathway involved in the regulation of cell differentiation, tissue polarity and cell proliferation (14). This pathway has been evaluated in the initiation and progression of several skin and gastrointestinal malignancies, such as esophageal and gastric cancer (15). It has been also shown to lead to adverse outcomes in patients with $\mathrm{HCC}$, including increased risk of tumor recurrence following surgical resection, and the increased expression of Gli1 has been suggested to be associated with poor prognosis in HCC (4). More recently, components of the hedgehog pathway have been targeted by specific inhibitors. For example, vismodegib, an inhibitor of the smoothened receptor, was the first hedgehog signaling pathway targeting agent to be approved by the Food and Drug Administration in early 2012 for the treatment of basal-cell carcinoma (16).

We documented the expression of Shh, Ptch and Gli1 proteins in human HCC and adjacent non-tumorous liver tissue by immunohistochemistry. Our results demonstrated that, among the Shh biomarkers, the expression level of Ptch was significantly higher in HCC compared with that in adjacent non-tumorous liver tissue. We observed a tendency for correlation of the level of Shh biomarker tissue expression in HCC with the recurrence rate following LT; however, this did not reach statistical significance. Those results may be used to perform power analysis calculations in future studies. While prior studies evaluated the association between Shh biomarkers and $\mathrm{HCC}$ recurrence following surgical resection $(4,17)$, to the best of our knowledge, this study is the first to report on correlating tissue expression of Shh biomarkers with HCC recurrence following LT.

There has been recent debate regarding the selection of HCC patients for LT, with arguments that the Milan criteria are too restrictive and that the selection criteria may be expanded without increasing the rate of HCC recurrence or compromising survival outcomes following LT. We consider that we may have more success in expanding the current LT selection criteria for HCC on the basis of the biological behavior of the tumor, rather than adjusting the number and size of tumors allowed. Several tumor biological factors such as DNA aneuploidy, high tumor cell proliferation index, high telomerase activity and mutation of the p53 gene, have been associated with increased risk of post-resection HCC recurrence (18-22). However, the prognostic impact of these factors remains uncertain following LT for HCC. Combining biomarkers that reflect tumor biology, such as hedgehog proteins, with clinical indicators of prognosis, such as the Milan criteria, is likely to more accurately predict HCC patient outcomes.

In conclusion, this proof-of-concept study has demonstrated higher levels of tissue expression of Ptch, among other Shh biomarkers, within HCC tumor compared with the surrounding non-tumorous liver tissue. The small sample size did not allow demonstrating an association between Shh biomarkers and HCC recurrence following LT. Further larger studies are required to assess the prognostic value of these biomarkers in HCC patients to fully elucidate their potential use in clinical practice. The present study is hypothesis-generating and further prospective analysis should be performed.

\section{Acknowledgements}

The present study was supported in part by the Mikati Foundation Endowed Chair in Liver Diseases (to Nizar Zein).

\section{References}

1. Shahi MH, Rey JA and Castresana JS: The sonic hedgehog-GLI1 signaling pathway in brain tumor development. Expert Opin Ther Targets 16: 1227-1238, 2012.

2. Parkin CA and Ingham PW: The adventures of sonic hedgehog in development and repair. I. Hedgehog signaling in gastrointestinal development and disease. Am J Physiol Gastrointest Liver Physiol 294: G363-G367, 2008.

3. Jeng KS, Sheen IS, Jeng WJ, Lin CC, Lin CK, Su JC, Yu MC and Fang HY: High expression of patched homolog-1 messenger RNA and glioma-associated oncogene-1 messenger RNA of sonic hedgehog signaling pathway indicates a risk of postresection recurrence of hepatocellular carcinoma. Ann Surg Oncol 20: 464-473, 2013.

4. Che L, Yuan YH, Jia J and Ren J: Activation of sonic hedgehog signaling pathway is an independent potential prognosis predictor in human hepatocellular carcinoma patients. Chin J Cancer Res 24: 323-331, 2012.

5. Parkin DM, Bray F, Ferlay J and Pisani P: Global cancer statistics, 2002. CA Cancer J Clin 55: 74-108, 2005.

6. El-Serag HB and Rudolph KL: Hepatocellular carcinoma: Epidemiology and molecular carcinogenesis. Gastroenterology 132: 2557-2576, 2007.

7. El-Serag HB and Mason AC: Rising incidence of hepatocellular carcinoma in the United States. N Engl J Med 340: 745-750, 1999.

8. Llovet JM, Burroughs A and Bruix J: Hepatocellular carcinoma. Lancet 362: 1907-1917, 2003.

9. Bruix J and Sherman M; Practice Guidelines Committee, American Association for the Study of Liver Diseases: Management of hepatocellular carcinoma. Hepatology 42: 1208-1236, 2005.

10. Macaron C, Hanouneh IA, Lopez R, Aucejo F and Zein NN: Total tumor volume predicts recurrence of hepatocellular carcinoma after liver transplantation in patients beyond Milan or UCSF criteria. Transplant Proc 42: 4585-4592, 2010.

11. Mazzaferro V, Regalia E, Doci R, Andreola S, Pulvirenti A, Bozzetti F, Montalto F, Ammatuna M, Morabito A and Gennari L: Liver transplantation for the treatment of small hepatocellular carcinomas in patients with cirrhosis. N Engl J Med 334: 693-699, 1996.

12. Toso C, Trotter J, Wei A, Bigam DL, Shah S, Lancaster J, Grant DR, Greig PD, Shapiro AM and Kneteman NM: Total tumor volume predicts risk of recurrence following liver transplantation in patients with hepatocellular carcinoma. Liver Transpl 14: 1107-1115, 2008.

13. Klintmalm GB: Liver transplantation for hepatocellular carcinoma: A registry report of the impact of tumor characteristics on outcome. Ann Surg 228: 479-490, 1998.

14. Ingham PW and McMahon AP: Hedgehog signaling in animal development: Paradigms and principles. Genes Dev 15: 3059-3087, 2001. 
15. Berman DM, Karhadkar SS, Maitra A, Montes De Oca R, Gerstenblith MR, Briggs K, Parker AR, Shimada Y, Eshleman JR, Watkins DN, et al: Widespread requirement for Hedgehog ligand stimulation in growth of digestive tract tumours. Nature 425: 846-851, 2003

16. Sekulic A, Migden MR, Oro AE, Dirix L, Lewis KD Hainsworth JD, Solomon JA, Yoo S, Arron ST, Friedlander PA, et al: Efficacy and safety of vismodegib in advanced basal-cell carcinoma. N Engl J Med 366: 2171-2179, 2012.

17. Zheng X, Yao Y, Xu Q, Tu K and Liu Q: Evaluation of glioma-associated oncogene 1 expression and its correlation with the expression of sonic hedgehog, E-cadherin and S100a4 in human hepatocellular carcinoma. Mol Med Rep 3: 965-970, 2010.

18. Tung-Ping Poon R, Fan ST and Wong J: Risk factors, prevention, and management of postoperative recurrence after resection of hepatocellular carcinoma. Ann Surg 232: 10-24, 2000.
19. Chiu JH, Kao HL, Wu LH, Chang HM and Lui WY: Prediction of relapse or survival after resection in human hepatomas by DNA flow cytometry. J Clin Invest 89: 539-545, 1992.

20. Ng IO, Lai EC, Fan ST, Ng M, Chan AS and So MK: Prognostic significance of proliferating cell nuclear antigen expression in hepatocellular carcinoma. Cancer 73: 2268-2274, 1994.

21. Suda T, Isokawa O, Aoyagi Y, Nomoto M, Tsukada K, Shimizu T, Suzuki Y, Naito A, Igarashi H, Yanagi M, et al: Quantitation of telomerase activity in hepatocellular carcinoma: A possible aid for a prediction of recurrent diseases in the remnant liver. Hepatology 27: 402-406, 1998.

22. Hayashi H, Sugio K, Matsumata T, Adachi E, Takenaka K and Sugimachi K: The clinical significance of p53 gene mutation in hepatocellular carcinomas from Japan. Hepatology 22: 1702-1707, 1995 